\title{
CONTENIDO DE GRASA Y PROTEINA EN Pygocentrus cariba, Prochilodus mariae, Plagioscion squamosissimus, Piaractus brachypomus e Hypostomus plecostomus EN UNA LAGUNA DE INUNDACION DEL ORINOCO MEDIO
}

\author{
Fat and Protein Content in Pygocentrus cariba, Prochilodus mariae, Plagioscion \\ squamosissimus, Piaractus brachypomus e Hypostomus plecostomus of a Middle \\ Orinoco Flood Lagoon
}

\author{
Ángel González ${ }^{1}$, Arístide Márquez², William Senior ${ }^{2}$ y Gregorio Martínez ${ }^{2}$ \\ ${ }^{1 * I n s t i t u t o ~ L i m n o l o ́ g i c o, ~ U n i v e r s i d a d ~ d e ~ O r i e n t e, ~ C a i c a r a ~ d e l ~ O r i n o c o, ~ E s t a d o ~ B o l i ́ v a r, ~(0284) 6667474, ~(0284) 6667090 . ~}$ \\ Cel. (0416)2477940. E-mail: angelgonzalez78@hotmail.com. 2Instituto Oceanográfico de Venezuela, Oceanografía Química, \\ Universidad de Oriente, Cumaná, Estado Sucre.
}

\begin{abstract}
RESUMEN
Se determinó la concentración de grasa y proteína en el tejido muscular de 40 peces de cada una de las especies Pygocentrus cariba, Prochilodus mariae, Plagioscion squamosissimus, Piaractus brachypomus e Hypostomus plecostomus, capturados en una laguna de inundación del rio Orinoco medio utilizando redes de enmalle. Los contenidos de proteína y grasa se determinaron por los métodos de la Asociación de Químicos Analíticos Oficiales (AOAC), estimándose un promedio en la concentración de grasa para $P$. cariba de $0,30 \pm 0,01 \%$; $0,60 \pm 0,01 \%$ para $H$. plecostomus; $0,65 \pm 0,01 \%$ para $P$. mariae; $0,90 \pm 0,01 \%$ para $P$. squamosissimus y $1,55 \pm 0,01 \%$ para $P$. brachypomus. El promedio en la concentración de proteína fue de $19,60 \pm 0,10 \%$ para $P$. mariae; $19,70 \pm 0,13 \%$ para $P$. squamosissimus; $19,70 \pm 0,09 \%$ para $H$. plecostomus; $19,80 \pm 0,15 \%$ para $P$. cariba y $19,80 \pm 0,15 \%$ para $P$. brachypomus. Un análisis de varianza (ANOVA) y prueba a posteriori de Duncan demostró diferencias estadísticamente significativas en el contenido de grasa entre especies, con los mayores valores correspondientes a $P$. brachypomus y $P$. squamosissimus y los menores a $P$. cariba. Los contenidos de proteína no presentaron diferencias significativas. Aparentemente las especies $P$. cariba, $P$. mariae, $P$. squamosissimus, $P$. brachypomus y $\mathrm{H}$. plecostomus, en general tienen un bajo contenido de grasa y una alta concentración de proteína, dependiendo sus concentraciones principalmente de la alimentación,
\end{abstract}

Recibido: 16 / 03 / 2007. Aceptado: 16/01/2008. época de reproducción y migración. El trabajo constituye un aporte al conocimiento de la composición química de estas especies, algunas de importancia comercial como $P$. squamosissimus, $P$. brachypomus y $P$. mariae y otras ecológicamente importantes como $P$. cariba y $H$. plecostomus.

Palabras clave: Peces de agua dulce, río Orinoco, composición química, grasa y proteína.

\begin{abstract}
It was determined the concentration of fat and protein in the muscular tissue of 40 fish of each one of the species Pygocentrus cariba, Prochilodus mariae, Plagioscion squamosissimus, Piaractus brachypomus and Hypostomus plecostomus; captured in a lagoon flood of the middle Orinoco river using gillnets. The protein content and fat were determined by the methods of the Association of Official Analytical Chemistry (AOAC), being considered an average in the concentration of fat for $P$. cariba of $0.30 \pm 0.01 \% ; 0.60 \pm 0.01 \%$ for $H$. plecostomus; $0.65 \pm$ $0.01 \%$ for $P$. mariae; $0.90 \pm 0.01 \%$ for $P$. squamosissimus and $1.55 \pm 0.01 \%$ for $P$. brachypomus. The average in the protein concentration was of $19.60 \pm 0.10 \%$ for $P$. mariae; $19.70 \pm$ $0.13 \%$ for $P$. squamosissimus $19.70 \pm 0.09 \%$ for $H$. plecostomus; $19.80 \pm 0.15 \%$ for $P$. cariba and $19.80 \pm 0.15 \%$ for $P$. brachypomus. A variance analysis (ANOVA) and a later test of Duncan it demonstrated differences statistically significant in the content of fat among species, with the bigger values of fat corresponding to $P$. brachypomus and $P$. squamosissimus and the smallest to $P$. cariba. The protein contents did not present significant differences. Seemingly the species $P$. cariba, $P$. ma-
\end{abstract}


Contenido de grasa y proteina en Pygocentrus cariba, Prochilodus mariae/ González, Á. y col.

riae, $P$. squamosissimus, $P$. brachypomus and $H$. plecostomus, in general have a low content of fat and a high protein concentration, depending their concentrations mainly of the food, reproduction time and migration. This work constitutes a contribution to the knowledge of the chemical composition of these species, some of commercial importance as $P$. squamosissimus, $P$. brachypomus and $P$. mariae and other ecologically important as $P$. cariba and $H$. plecostomus.

Key words: Freshwater fish, Orinoco River, chemical composition, fat and protein.

\section{INTRODUCCION}

La composición química de los peces varía entre diferentes especies y en una misma especie, según su edad, sexo, ambiente y época del año [11]. Específicamente los contenidos de grasa y proteína están estrechamente relacionados con la alimentación, la migración y los cambios sexuales asociados con el desove, como consecuencia de los períodos de inanición que frecuentemente presentan. La migración y el desove generalmente ocasionan un gasto de la energía que tienen los peces almacenada en forma de grasa mientras que aquellas especies que realizan largas migraciones, también pueden utilizar las reservas de proteína, además de las reservas de grasa, como fuente de energía [4, 6, 17, 20].

Después de la migración y el desove, los peces recuperan el contenido de proteína a través del alimento consumido, a la vez que aumentan notablemente el contenido de grasa [31]. Este parámetro es el que muestra generalmente una mayor variación anual con relación al contenido de proteína, con una curva estacional característica que frecuentemente presenta un mínimo cuando se acerca la época del desove. La acumulación de grasa y proteína constituye por lo tanto, un aspecto esencial para el éxito ecológico de los peces, especialmente para aquellos que usan las reservas de energía para la migración y la reproducción [19, 31].

En el presente trabajo se hizo un análisis del contenido de grasa y proteína en el tejido muscular del caribe colorado Pygocentruss cariba, el coporo Prochilodus mariae, la curvinata de río Plagioscion squamosissimus, el morocoto Piaractus brachypomus y la guaraguara o corroncho Hypostomus plecostomus; como un aporte al conocimiento de su composición química y la posible relación del contenido de grasa y proteína con la alimentación, migración y reproducción, sin descartar la contribución de los resultados, como información para los nutricionistas, tecnólogos de alimentos y acuicultores, interesados en la evaluación de aquellos recursos con bajos contenidos de grasa y alto contenido proteico [19].

\section{MATERIALES Y MÉTODOS}

El trabajo se realizó con 40 ejemplares de las especies $P$. cariba $(19,0-27,0 \mathrm{~cm}$ de longitud total), P. mariae $(28-32 \mathrm{~cm}$ de longitud total), $P$. squamosissimus $(30-38 \mathrm{~cm}$ de longitud total), $P$. brachypomus $(23-31 \mathrm{~cm}$ de longitud total) e $H$. plecostomus (30 $-40 \mathrm{~cm}$ de longitud total), capturados en la laguna de inundación Castillero (7³9'09" N y 6609'00" W) de la región de Caicara del Orinoco, estado Bolívar, Venezuela. Los ejemplares fueron capturados entre los meses de noviembre 1998 y marzo 1999, época de sequía, utilizando chinchorros de enmalle de $10 \mathrm{~cm}$ de abertura de malla y posteriormente trasladados en cavas isotérmicas hasta el laboratorio, donde fueron mantenidos a $20^{\circ} \mathrm{C}$ hasta el momento de su procesamiento.

Durante los meses de lluvia e inundaciones disminuyó notablemente la accesibilidad de las especies estudiadas hacia el arte de pesca utilizado durante los muestreos y hacia otros tipos de artes, imposibilitándose el análisis de las especies para estos meses por insuficiencia de datos. Igualmente, durante los meses de sequía, el número de peces capturados fue insuficiente para la estratificación del muestreo por sexo, madurez sexual y para todos los rangos de talla; considerándose en el análisis una muestra constituida por 40 ejemplares de cada especie, sin diferenciación de sexo y madurez sexual, con rangos cortos de tallas y capturados durante la época de sequía, previa demostración de diferencias no significativas en el contenido de grasa y proteína durante los meses muestreados.

Cada ejemplar, de cada especie, fue medido individualmente y después fileteado para tomar muestras del tejido muscular y hacer el análisis, realizándose un total de 40 análisis por especie. Antes del análisis, la cabeza, cola, aletas, vísceras, y piel de cada ejemplar fueron retiradas, obteniéndose los filetes cortando cuidadosamente el tejido muscular a lo largo de la línea lateral de cada ejemplar [19].

Los porcentajes de proteínas y grasas se determinaron por los métodos de la Asociación de Químicos Analíticos Oficiales (AOAC) [1]. Las grasas crudas se extrajeron por reflujo (AOAC.920.29) con éter dietílico purificado, utilizando balones de Pyrex (EUA) de $250 \mathrm{~mL}$ de capacidad, adjuntados a un sistema de tubos refrigerantes. El éter fue luego evaporado a $105^{\circ} \mathrm{C}$ en una estufa marca Memmert (Alemania), determinándose los porcentajes posteriormente por diferencia de peso [1].

El contenido de proteína fue determinado por el método Kjeldah de la Asociación de Químicos Analíticos Oficiales [1], el cual consiste en la digestión de muestras del tejido muscular de los peces $(0,3-0,4 \mathrm{~g})$ en $25 \mathrm{~mL}$ de ácido sulfúrico concentrado, junto con $0,7 \mathrm{~g}$ de óxido de mercurio y $15 \mathrm{~g}$ de sulfato de potasio. Después de la digestión, una solución de $250 \mathrm{~mL}$ de agua y $25 \mathrm{~mL}$ de sulfuro de potasio al $4 \%$, junto con una solución de $50 \mathrm{~mL}$ de hidróxido de sodio, fueron añadidas a las muestras digeridas. El amoníaco fue destilado en ácido bórico al $2 \%(25 \mathrm{~mL})$ y titulado con ácido hidroclórico $0,1 \mathrm{~N}$; utilizando rojo de metilo-verde bromocresol como indicador. La proteína fue calculada multiplicando el valor del nitrógeno por 6,25 [19].

Los promedios de los contenidos de grasa y proteína de las especies consideradas fueron comparados estadísticamen- 
te a través de un análisis de varianza (ANOVA) y prueba a posteriori de Duncan [13].

\section{RESULTADOS Y DISCUSIÓN}

El promedio de la concentración de grasa en $P$. cariba fue de $0,30 \pm 0,01 \%$, el de $P$. mariae $0,65 \pm 0,01 \%$, el de $P$. squamosissimus $0,90 \pm 0,01 \%$, el de $P$. brachypomus $1,55 \pm 0,01 \%$ y el de $H$. plecostomus $0,60 \pm 0,01 \%$; con diferencias estadísticamente significativas en las concentraciones de grasas $(\mathrm{P}=0,0000 ; \alpha=0,05)$. Las especies con mayor concentración de grasa fueron $P$. brachypomus y $P$. squamosissimus, sin diferencias significativas entre sí, seguido de las especies $P$. mariae, $H$. plecostomus, también sin diferencias significativas entre sí, y por último $P$. cariba con la menor concentración (TABLA I). Esta variación en el contenido de grasa entre especies generalmente está determinada por el efecto diferencial de algunos factores ambientales, alimenticios y fisiológicos [11, 17].

El promedio en la concentración de proteína de $P$. cariba fue de $19,80 \pm 0,15 \%$, el de $P$. mariae $19,60 \pm 0,10 \%$, el de $P$. squamosissimus $19,70 \pm 0,13 \%$, el de $P$. brachypomus $19,80 \pm$ $0,15 \%$ y el de $H$. plecostomus $19,70 \pm 0,09 \%$, sin diferencias significativas entre ellas (TABLA II). En cada especie se observó una alta homogeneidad en las concentraciones de grasa y proteína entre los ejemplares analizados, contrario a lo que se esperaría si se toma en cuenta el carácter heterogéneo de las muestras en cuanto al sexo, madurez sexual y meses de captura. Esta aparente homogeneidad posiblemente estuvo relacionada con el corto rango de tallas de los peces analizados (peces de tamaño aproximadamente iguales), así como con la época específica de sequía cuando fueron capturadas $[11,17]$.

El rango de variación del contenido de grasa en los peces está entre 0,2 y $25 \%$ mientras que particularmente en los peces de agua dulce el rango varía entre 0,6 y $14 \%[11,17$, 29]. En las especies tropicales de agua dulce el contenido de grasa rara vez pasa del 5\% [11] como se demostró en el presente trabajo, donde el contenido de grasa varió entre 0,28 y $1,56 \%$, e igualmente, en otras especies que se encuentran en lagunas de inundación del Orinoco como Pseudoplatystoma fasciatum, donde el contenido de grasa ha sido estimado en $0,85 \%$ [16]. En algunas especies de agua dulce de la India el contenido de grasa ha sido determinado entre 0,6 y 1,3\% [7].

El contenido de proteína, al igual que en la mayoría de los peces de agua dulce, fue relativamente alto $(19,60-19,80 \%)$ y estuvo ubicado dentro del rango señalado para los peces en general $(0,2-25 \%)$ y para los peces de agua dulce (17 -22\%) $[11,17,29]$. En el Orinoco se ha determinado un contenido de proteína en $P$. fasciatum de 18,1\% [16], mientras que en algunas especies de la India se ha estimado ente 17 y $22 \%$ [7].

En muchas especies, como algunas de agua dulce de la Argentina, los contenidos de grasa y proteína están correlacionados entre sí [18] e igualmente relacionados con el tamaño de los peces en general $[8,11,17]$. Estas relaciones no se observaron en las especies aquí estudiadas, aunque el pequeño rango de tamaño de los ejemplares analizados pudo influir en el resultado de la relación con el tamaño. Sin embargo, en algunas especies de agua dulce del noreste de los Estados Unidos, también se ha demostrado no haber relación entre el contenido de grasa y proteína con el tamaño de los peces [19].

Considerando los resultados por especies, $P$. brachypomus fue la que presentó un mayor contenido de grasa $(1,55 \%)$ junto con $P$. squamosissimus $(0,90 \%)$ (TABLA I), así como un alto contenido de proteína $(19,80 \%)$ (TABLA II) al igual que todas las especies analizadas. Se trata de una especie omnívora, predominantemente herbívora, que se alimenta de diferentes hojas, flores, frutos y semillas durante sus fases de juvenil y adulto [3, 21, 25], aunque en el Orinoco, principalmente durante la época de sequía, tiende a consumir otros tipos de alimentos como insectos acuáticos, algunos invertebrados bentónicos y otros recursos de origen animal ricos en proteína [25, 28], los cuales posiblemente influyeron en su alto contenido proteico [26, 27].

El tamaño de los peces $P$. brachypomus utilizados en el análisis varió entre 23 y $31 \mathrm{~cm}$ de longitud total y por lo tanto, como son considerados peces inmaduros, posiblemente el contenido de grasa y proteína fue independiente de la madura-

TABLA I

PRUEBA DE RANGO MULTIPLE (DUNCAN) PARA EL CONTENIDO DE GRASA (\%) EN EL TEJIDO MUSCULAR DE PECES DEL ORINOCO MEDIO DE VENEZUELA/ TEST OF MULTIPLE RANGE (DUNCAN) FOR THE CONTENT OF FAT (\%) IN THE MUSCULAR TISSUE OF THE MIDDLE ORINOCO FISH IN VENEZUELA.

\begin{tabular}{lccccc}
\hline \multicolumn{1}{c}{ Especies } & $\mathrm{N}$ & Rango & $\times$ & D.E & G.H \\
\hline P. cariba & 40 & $0,28-0,32$ & 0,30 & 0,01 & X \\
H. plecostomus & 40 & $0,58-0,62$ & 0,60 & 0,01 & X \\
P. mariae & 40 & $0,63-0,67$ & 0,65 & 0,01 & X \\
P. squamosissimus & 40 & $0,89-0,91$ & 0,90 & 0,01 & $X$ \\
$P$. brachypomus & 40 & $1,54-1,56$ & 1,55 & 0,01 & $X$ \\
\hline
\end{tabular}

$\mathrm{N}=$ Tamaño de la muestra.

$x=$ Promedio.

D.E.= Desviación estándar.

G.H. $=$ Grupos homogéneos. 
Contenido de grasa y proteina en Pygocentrus cariba, Prochilodus mariae/ González, Á. y col.

TABLA /I

PRUEBA DE RANGO MULTIPLE (DUNCAN) PARA EL CONTENIDO DE PROTEINA (\%) EN EL TEJIDO MUSCULAR DE PECES DEL ORINOCO MEDIO DE VENEZUELA/ TEST OF MULTIPLE RANGE (DUNCAN) FOR THE CONTENT OF PROTEIN (\%) IN THE MUSCULAR TISSUE OF THE MIDDLE ORINOCO FISH IN VENEZUELA.

\begin{tabular}{lccccc}
\hline \multicolumn{1}{c}{ Especies } & $\mathrm{N}$ & Rango & $\times$ & D.E. & G.H. \\
\hline P. mariae & 40 & $19,4-19,8$ & 19,60 & 0,10 & $X$ \\
P. squamosissimus & 40 & $19,5-19,8$ & 19,70 & 0,13 & $X$ \\
H. plecostomus & 40 & $19,5-19,9$ & 19,70 & 0,09 & $X$ \\
P. cariba & 40 & $19,3-20,0$ & 19,80 & 0,15 & $X$ \\
P. brachypomus & 40 & $19,3-20,0$ & 19,80 & 0,15 & $X$ \\
\hline
\end{tabular}

$\mathrm{N}=$ Tamaño de la muestra.

$x=$ Promedio .

D.E.= Desviación estándar.

G.H. = Grupos homogéneos.

ción sexual que ocurre durante los meses de sequía, manteniendo relativamente alto su contenido de grasa. Probablemente el contenido de grasa disminuya en los adultos durante la época de maduración sexual, mientras que el alto contenido de proteína se conserve debido principalmente a que las migraciones que la especie realiza para el desove, además de ser cortas y transversales, son discontinuas e interrumpida ocasionalmente para alimentarse y recuperar energía [23]. El contenido de proteína de $P$. brachypomum en el Amazonas es de 15,8\% [2] y el de grasa está comprendido entre menos de $1,5 \%$ [2] y $15,6 \%$ [17]. Estos valores son diferentes a los estimados para el Orinoco medio, debido posiblemente a factores relacionados con el tamaño, tipo de alimento, sexo, madurez sexual y época de captura.

En la especie $P$. cariba, el contenido de grasa fue el más bajo de las especies analizadas con un 0,30\% (TABLA I), mientras que el contenido de proteína fue alta (19,8\%) (TABLA II). La longitud de los peces estuvo comprendida entre 19 y $27 \mathrm{~cm}$ de longitud total y aparentemente ya maduros sexualmente [14], por lo que el bajo contenido de grasa posiblemente estuvo asociado con el consumo de energía para la maduración y el desove, considerando que la especie madura durante los últimos meses de sequía y desova justamente antes de la época de lluvia [28]. Durante la época de lluvia del Orinoco el contenido de grasa en $P$. cariba es de $6,61 \%$ y de proteína $17,9 \%$ [30], mayores que los estimados para la época de sequía. Además de la época de reproducción, otros factores como el sexo y el tamaño pudieron haber influidos en esta diferencia.

$P$. cariba, al igual que $P$. brachypomum, presenta una marcada plasticidad en sus hábitos alimenticios y aprovecha en determinadas circunstancias la disponibilidad de cualquier tipo de alimento incluyendo vegetales, aunque es una especie preferentemente carnívora $[2,15,22,27]$. Su alto contenido de proteína posiblemente lo determina el tipo de alimento animal que preferiblemente consume [26, 27], el cual probablemente no es afectado por factores como la migración, por cuanto ésta es relativamente corta y transversal $[23,29]$.
En $P$. mariae, al igual que en $H$. plecostomus, el contenido de grasa fue medianamente baja $(0,65 \%)$ (TABLA I), mientras que la concentración de proteína también fue relativamente alta $(19,60 \%)$ (TABLA II). La talla de los ejemplares estuvo comprendida entre 28 y $32 \mathrm{~cm}$ de longitud total y por lo tanto con edad de reproducirse [9]. En el río Unare (Venezuela) se ha determinado una concentración de grasas y proteínas en $P$. mariae de 3,67 y $19,03 \%$, respectivamente, durante la fase de reposo gonadal (enero, febrero y marzo); 1,24 y 18,99\%, respectivamente, durante la fase de desarrollo gonadal (junio, julio) y 0,18 y $17,44 \%$, respectivamente, durante el post-desove (julio, agosto y septiembre) [4]. Aproximadamente, el contenido de grasa y proteína estimada en el Unare durante la fase de desarrollo gonadal coincidió con los determinados para el Orinoco medio durante la época del muestreo, por lo que posiblemente las gónadas de $P$. mariae en la región durante los últimos meses de sequía igualmente estaban en su fase de desarrollo, considerando que su máxima intensidad reproductiva en el Orinoco ocurre durante los primeros meses de lluvia [28]. Durante la época de reproducción, la proteína de $P$. mariae generalmente es utilizada como energía durante las largas migraciones que realiza a lo largo del canal principal para el desove [32], aunque en este caso se mantuvo el contenido de proteína por cuanto posiblemente en la región del Orinoco medio, la especie no necesita realizar largas migraciones para desovar por cuanto puede hacerlo en lagunas como la de Castillero [31].

En la especie $H$. plecostomus, al igual que en $P$. mariae, el contenido de grasa fue medianamente bajo $(0,6 \%)$ (TABLA I) mientras que la concentración de proteína fue relativamente alto $(19,70 \%)$ (TABLA II). La talla de los peces estuvo comprendida entre 30 y $40 \mathrm{~cm}$ de longitud total y aparentemente con posibilidad de haber alcanzado la primera madurez sexual [23]. Probablemente la madurez sexual y el desove influyeron en el bajo contenido de grasa estimado para la especie, por cuanto éstas ocurren durante los últimos meses de sequía e inicio de las lluvias [25]. Los contenidos de grasa y proteína estimados para el Orinoco medio coinciden aproximadamente con los determinados para otros ríos suramericanos $(0,7 \%$ de grasa y $20,3 \%$ de 
proteína) [32], mientras que en algunas regiones centroamericanas como El Infiernillo (México) las concentraciones son muy superiores $(12,13 \%$ de grasa y $85 \%$ de proteína) [10]. Estas diferencias pudieron estar relacionadas con variaciones en el tamaño, sexo, madurez y época de captura. El alto contenido de proteína de $H$. plecostomus, junto con su abundancia y escaso valor comercial y como especie de consumo en la región del Orinoco medio, son aspectos importantes para considerarla como un posible potencial ingrediente en la formulación de dietas balanceadas forrajeras, como se ha recomendado para el reservorio El Infiernillo en México [10].

Los peces $P$. squamosissimus estuvieron comprendidos entre 30 y $38 \mathrm{~cm}$ de longitud total y por lo tanto ya con edad de madurez sexual [24]. Sin embargo su contenido de grasa fue relativamente alto $(0,9 \%)$ (TABLA I), al igual que $P$. brachypomus, además de presentar también un alto contenido de proteína de 19,70\% (TABLA II). Es una especie netamente carnívora que se alimenta de peces y camarones de agua dulce [12] pero que también puede consumir en su etapa juvenil microcrustáceos (larvas de camarones, copépodos) e insectos acuáticos [28], los cuales son ricos en proteína [26, 27]. Para el Orinoco existen en $P$. squamosissimus dos períodos reproductivos al año, abril - julio y septiembre - diciembre [28], por lo que posiblemente para la época del muestreo (principalmente durante los meses de enero, febrero y marzo) los peces ya habían desovado y se encontraban en estado de reposo gonadal, acumulando energía en forma de grasa. En el Amazonas, el desove de $P$. squamosissimus ocurre durante todo el año sin picos reproductivos bien definidos [10], lo que implica probablemente una continua acumulación de grasa que alcanza valores de $1,02 \%$ [5], aproximadamente igual al estimado para el Orinoco medio y aún superiores (5,9\%) [17].

\section{CONCLUSIONES Y JUSTIFICACIONES}

Las especies continentales Pygocentrus cariba, Prochilodus mariae, Plagioscion squamosissimus, Piaractus brachypomus e Hypostomus plecostomus tienen bajo contenido de grasa y altas concentraciones de proteína, con variaciones entre especies en el contenido de grasa relacionadas principalmente con la época de reproducción y los movimientos migratorios de cada una de ellas. Estos resultados podrían resultar valiosos para los nutricionistas y tecnólogos de alimentos, en su afán de evaluar la disponibilidad de recursos de bajo contenido de grasa y alto contenido proteico. Igualmente para los acuicultores interesados en el conocimiento de la composición nutritiva de los peces de agua dulce con fines de cultivo.

Los bajos contenidos de grasa y alta concentración de proteína les proporciona a estas especies un alto valor nutritivo como alimento para el venezolano, debiéndose incentivar el consumo de especies como $P$. cariba, $P$. mariae y $H$. plecostomus, además por su escaso valor comercial y abundancia en el Orinoco. Incluso $H$. plecostomus podría utilizarse como in- grediente de dietas balanceadas forrajeras por su alto contenido proteico, abundancia y poco consumo.

\section{AGRADECIMIENTO}

Al Fonacit (Proyecto Pem-2001001628) y Fundacite Guayana (Proyecto 000606), así como al Consejo de Investigación de la Universidad de Oriente, por el financiamiento de los proyectos que dieron origen a la presente investigación. Igualmente al Instituto Limnológico de la Universidad de Oriente por la logística prestada.

\section{REFERENCIAS BIBLIOGRÁFICAS}

[1] ASSOCIATION OF OFFICIAL ANALYTICAL CHEMIST (AOAC). 14 th Ed. Horwitz, W. De. (Ed). Washington. 1141 pp. 1997.

[2] ARAUJO-LIMA, C.; GOULDING, M. So Fruitful a Fish: Tambaqui, Aquaculture and Conservation in the Amazon. Columbia University Press: New York. 157 pp. 1997.

[3] ARAUJO-LIMA, C.; RUFINO, M.L. Migratory Fishes of the Brazilian Amazon. In: Carolsfeld, J.; Harvey, B.; Ross, C.; Baer, A. (Eds). Migratory Fishes of South America: Biology, Fisheries and Conservation Status. Victoria BC: The World Bank/IDRC/World Fisheries Trust. Pp 235-301. 2003.

[4] ARVELAIZ, P. V.; BELLO, R. Cambios en las propiedades químicas y bioquímicas del músculo del coporo (Prochilodus mariae) asociados al ciclo reproductivo. Rev. Científ. FCV-LUZ. XV (4): 368 - 376. 2005.

[5] BELLO, R. A.; RIVAS, W. G. Evaluación y aprovechamiento de la cachama Colossoma macropomum cultivada como fuente de alimento. Proyecto Aquila II, FAO, México. 113 pp. 1992.

[6] BURGES, W.E. A preliminary survey of the Siluriformes. An atlas of freshwater and marine catfishes. T.F.H. Publications, Inc., Neptune City, New Jersey (USA). 1-784 pp. 1989.

[7] CHANDRASHEKAR, K.; DEOSTHALE, Y.G. Proximate composition, amino acid, mineral, and trace element content of the muscle of 20 Indian fish species. J. Food Comp. and Anal. 6(2): 195-200. 1993.

[8] DE OLIVEIRA, E.R.N.; AGOSTINHO, A.A.; MATSUSHITA, M. Effect of biological variables and capture period on the proximate composition and fatty acid composition of the dorsal muscle tissue of Hypopthalmus edentatus (Spix,1829). Braz. Arch. Biol. Technol. 46(1): 105-114. 2003. 
Contenido de grasa y proteina en Pygocentrus cariba, Prochilodus mariae/ González, Á. y col

[9] DUQUE, A.B.; TAPHORN, D.C.; WINEMILLER, K.O. Ecology of the coporo Prochilodus mariae (Characiforme, Prochilodontidae), and status of annual migrations in western Venezuela. Environm. Biol. Fishes. 53(1): 33-46. 1998.

[10] ESCALERA-GALLARDO, C; ARROLLO-DAMIAN, M.; ZUNO-FLORIANO, F. Physicochemical characterization of the invasive species Hypostomus plecostomus and alternatives for its use in Mexico. CIIDIR-Instituto Politécnico Nacional México y Baylor University, Waco, TX, USA. International Conference, Mérida, México, Enero 12, 2006. En línea: http://abstracts.co.allenpress .com/pweb /esai2006/document/?ID=58573. 01 de Febrero 2007.

[11] ESPEJO - HERMES, J. Composition of fish. In: Manual on fish handing and processing. Cambodia proyect on standards, quality and conformity assessment. European Union-Cambodia Co-operation. Proyect number: European Aid/120277/C/SV/KH. 66 pp. 2006.

[12] FELDEBERG, E.; REBELO, J.I.; PEDRACA DOS SANTOS, E.B.; SOUZA V, F.C. Cytogenetic studies of two freshwater sciaenids of the genus Plagioscion (Perciforme, Sciaenidae) from the central Amazon. Genet. Mol. Biol. 22(3): 351-356. 1999.

[13] FERNÁNDEZ, P. F.; LÓPEZ, S. M. A.; MUÑOZ, M. M.; RODRIGUEZ, C. A. M.; SÁNCHEZ, N. A.; VALERO, F. C. Estadística asistida por ordenador. Statgraphics Plus 4,1. Universidad de Cadiz, Servicio de Publicaciones, España, 2004. En línea: http://www2. uca.es/grup-invest/teloydisren/alumnus/eapo/eapo.htm-2k. 05 de marzo 2007.

[14] FINK, W. L. Revision of Piranha Genus Pygocentrus (Teleostei, Characiformes). Copeia. 1993(3): 665687. 1993.

[15] GONZÁLEZ, A.; GONZÁLEZ, E. Variación Estacional y Ontogenética en la Alimentación del Caribe Colorado Pygocentrus cariba, en una Laguna de Inundación de la región del Orinoco Medio. SABER. UDO. 13(1): 3-7. 2001.

[16] GONZÁlEZ, A.; MÁRQUEZ, A.; SENIOR, W.; MARTINEZ, G. Concentración de K, Na, Ca, Mg, Fe, proteínas y grasas en el bagre rayado Pseudoplatystoma fasciatum del Orinoco medio en Venezuela. Rev. Tec. Ing. Univ. Zulia. 29(2): 119-126. 2006.

[17] HUSS, H.H. Quality and quality changes in freshwater fish. FAO Fish. Techn. Paper No. 348. 203 pp. 1995.
[18] JUÁREZ, M.P. Body composition of Argentina freshwater fishes. A mathematical approach to the chemical composition of Oligosarcus jenynsi (Characdidae). Comp. Biochem. and Physiol. Part A: Physiology. 81(3): 473-479. 1985.

[19] KINSELLA, J.E.; SHIMP, J.L.; MAI, J. The proximate and lipid composition of several species of freshwater fishes. N.Y. Food Life Sci. No. 69. 20 pp. 1978.

[20] LARSON, R.J. Seasonal cycles of reserves in relation to reproduction in Sebastes. Environ. Biol. Fishes. 30 (1-2): 57-70. 1990.

[21] LOUBENS, G. Biologie de Piaractus brachypomus (Teleostei: Serrasalmidae) dans le bassin du Mamoré (Amazonie bolivienne). Ichthyol. Explor. Freshwat. 12(1):51-64. 2001.

[22] MACHADO-ALLISON, A.; FINK, W. L. Sinopsis de las especies de la subfamilia Serrasalminae presentes en la cuenca del Orinoco. Claves, diagnosis e ilustraciones. Series Peces de Venezuela. Universidad Central de Venezuela. 90 pp. 1995.

[23] MANNHEIMER, S.; BEVILACQUA, G.; CARAMASCH, E. P.; SCARANO, F. R. Evidence for seed dispersal by the catfish Auchenipterichthys longimanus in an Amazonian lake. J. Trop. Ecol. No. (19): 215-218. 2003.

[24] [24] LOUBENS, G. Biologie de Plagioscion squamosissimus (Teleostei: Scianidae) dans le basin du Mamoré (Amazonie Bolivienne). Ichthyol. Explor. Freshwat. 14 (4): 335 - 352. 2003.

[25] MERONA, B.; RANKIN, M, de J. Food resource partitioning in a fish community of the central Amazon flooplain. Neotrop. Ichthyol. 2(2): 75-84. 2004.

[26] NICO, L.G.; MORALES, M. de. Nutrient content of piraña (Characidae, Serrasalminae) prey items. Copeia. 1994(2): 524-528. 1994.

[27] NICO, L.G.; TAPHORN, D.C. Those bitin fish from South America. Trop. Fish Hobbyst. 34(4): 24-57. 1986.

[28] NOVOA, R.D.F. Los recursos pesqueros del eje fluvial Orinoco-Apure: Presente y Futuro. Ministerio de Agricultura y Tierra, INAPESCA, Venezuela. 148 pp. 2002.

[29] PUWASTIEN, P.; JUDPRASONG, K.; KETTWAN, E.; VASANACHIIT, K.; NAKNGAMANONG, Y.; BHATTACHARJEE, L. Proximate Composition of raw and cooked thai freshwater and marine fish. J. Food Comp. Analysis. 12(1): 9-16. 1999. 
[30] RODRIGUEZ, R. J. C.; CABELLO, A.M.; FIGUERA, B.; RAMOS, M.; BALLENILLA, O. Caracterización y aprovechamiento de la pulpa del caribe colorado Pygocentrus cariba. Intercien. 26(4): 161-165. 2001.

[31] SALDAÑA, J.; VENABLES, B. Energy compartmentalization in a migratory fish, Prochilodus mariae (Proci- lodontidae), of the Orinoco river. Copeia. 1983 (3): 617-623. 1983.

[32] WINEMILLER, K.O.; JEPSEN, D.B. Effects of seasonality and fish movement on tropical river food webs. J. Fish Biol. 53(supplement A): 267-296. 1998. 